\title{
Voice Perturbations in Repaired Cleft Lip and Palate
}

\author{
Navya Attuluri*, Pushpavathi M, Preeti Pandey and Spruha Mahapatra \\ AIISH, Mysore, India.
}

Submission: May 04, 2017; Published: May 24, 2017

*Corresponding author: Attuluri Navya, AIISH, Mysore, 48, First Floor, Maruthi temple road, T.K. Layout, Kuvempunagar, Mysore, India, Tel: 9036377964; Email: navyaaslp@gmail.com

\begin{abstract}
Context: The speech of individuals with cleft lip and palate (CLP) is dominated by articulatory, resonatory and voice abnormalities. The research on voice abnormalities in CLP is not extensively studied despite high prevalence (9.2\%) of hoarse voice in children with CLP.

Aims: The current study is aimed at exploring the salient features of voice in children with CLP. The primary objective is to evaluate the jitter, shimmer and harmonic noise ratio measures in children with repaired cleft lip and palate (RCLP), age and gender matched typically developing children (TDC). The second objective is to compare the voice measures of RCLP with TDC.

Settings and Design: The study is conducted in a clinical setting using standard group comparisons design.

Methods and Material: The study included 49 participants (17 RCLP and 32 TDC) in the age range of 7 years to 13 years. The phonation of vowel /a/ and /i/ were recorded and considered as speech samples for voice evaluation. The PRAAT 5.1 software is used for acquiring local jitter\%, local shimmer\% and harmonic to noise ratio (HNR) measures.
\end{abstract}

Statistical analysis used: Multivariate analysis is performed using SPSS Statistical Base 17.0 version was used.

Results: The results indicated increased jitter and shimmer measures and reduced HNR measures in children with RCLP than TDC. However, the statistically significant differences were noticed only for jitter $(\mathrm{P}<0.05)$ for the vowel $/ \mathrm{a} /$.

Conclusion: The deviant spectral measures indicate that children with RCLP are prone to develop voice disturbance and need to be evaluated.

Keywords: Jitter; Shimmer; Perturbations; Voice; Cleft Lip and Palate

\section{Introduction}

The children with cleft lip and palate (CLP) often exhibit various speech abnormalities due to structural anomalies. There are extensive studies [1,2] on evaluating the articulation and resonance aspects of speech and very few studies focused on voice parameters in children with CLP. Hocevar-Boltezar, Jarc, and Kozelj [3] reported that $9.2 \%$ of children with CLP are having hoarse voice secondary to the functional voice disorder.

Researchers have reported that children with CLP may exhibit hoarseness, breathiness, reduced loudness, and hypernasality [4]. Some of the authors tried to provide possible explanations for the abnormal voice characteristics. In the presence of velopharyngeal dysfunction, children with RCLP try to compensate for the loss of air pressure by increasing respiratory effort in order to build adequate intraoral pressure. This is because they need extra effort to attain normal intensity level due to acoustic damping in the nasal tract [5]. The children develop hoarseness as a compensatory mechanism to reduce the perception of hypernasality. Actually, when a person speaks with a hoarse voice, he/she might be using the glottis as a part of the articulation organ, and this might cause a spectral change. The presence of glottal stop in the speech of children with cleft lip and palate is widely reported [6,7].

It has been reported by Warren et al. [8] that increased respiratory effort can lead to vocal abuse which is most often seen in individuals with VPI. Specifically, for nasalized vowels opening phase of the vocal fold will be reduced due to altered vocal cord vibrations as reported by Hamlet [9]. Voice is evaluated by studying the various parameters like fundamental frequency, intensity and perturbation measures such as jitter and shimmer. Jitter is defined as the parameter of frequency variation from cycle to cycle, and shimmer relates to the amplitude variation of the sound wave [10]. In Figure 1 it can be seen the representation of these parameters. These parameters can be analyzed under a steady voice producing a vowel continuously. 


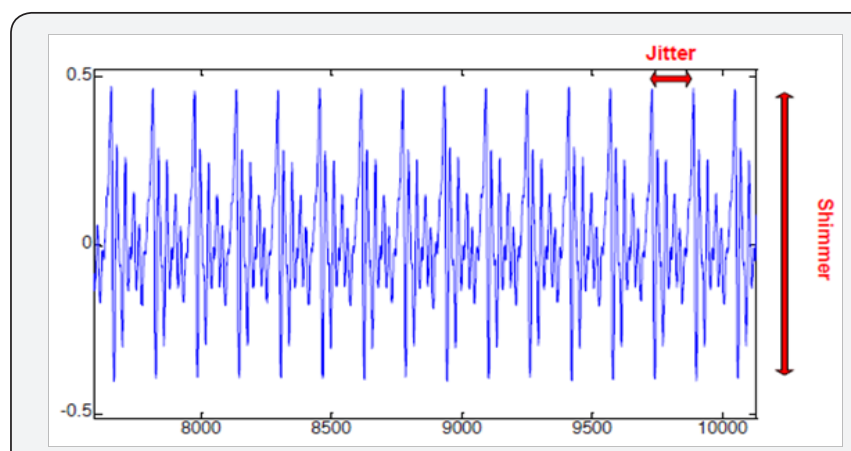

Figure 1: Representation of Jitter and Shimmer perturbation measures in speech signal.

The jitter is affected mainly by the lack of control of vibration of the cords; the voices of patients with pathologies often have a higher percentage of jitter. Most researchers considered as typical value variation between 0.5 and $1.0 \%$ for the sustained phonation in young adults. The shimmer changes with the reduction of glottal resistance and mass lesions on the vocal cords and is correlated with the presence of noise emission and breathiness. It is considered pathological voice for values less than $3 \%$ of adults and around 0.4 and $1 \%$ for children $[10,11]$. Zajac and Linville [4] explored the voice perturbations in 10 children (6.8 to 11.10 years) with VPI exhibiting perceived nasality and hoarseness using electroglottography. The results indicated a positive correlation between the jitter measures and perceived nasality. Whereas perceived hoarseness was positively correlated with shimmer measures.

The abnormal voice quality exhibits variations in perturbation measures. In addition to these Yumoto, Gould \& Baer [12] indicated that the degree of hoarseness in voice can also be evaluated by judging the extent of noise replacing the harmonic structure in the spectrum of a sustained vowel. Sustained vowels are perceived as hoarse having noise components in the main formants of various vowels. The normal voice shows a regular harmonic structure up to about $4 \mathrm{KHz}$ with relatively low noise energy. In the case of the pathologic voice, the spectrum shows higher noise levels with deterioration harmonic stricter even at lower frequencies. The harmonics-to-noise energy ratio (HNR) at different frequency bands can, therefore, be used for discriminating pathologic voices from normal ones. Harmonic to noise ratio is defined as the ratio between periodic components and non periodic component comprising a segment of voiced speech, as Murphy and Akande [13]. A low HNR denotes an asthenic voice and dysphonia. That is, with a value of less than 7 $\mathrm{dB}$ in HNR is considered pathological, as Boersma [14].

Hence, it is essential to evaluate the salient features of voice using various subjective and objective measures. The perceptual evaluation is often used [15] and objective measures HNR, jitter and shimmer were not explored widely in children with CLP. Hence, evaluation of these voice parameters provides us insight for better understanding and diagnosing the speech difficulties of children with cleft lip and palate. The study aimed at exploring the salient features of voice in children with repaired cleft lip and palate. The first objective of the study is to evaluate the perturbation measures (jitter and shimmer), and harmonic to noise ratio (HNR) in children with RCLP and TDC. The study also compared these measures across the groups and stimuli.

\section{Subjects and Methods}

\section{Research design: Standard Group comparison design is used.}

\section{Selection and Description of Participants}

The study included a total of 49 participants using convenience sampling method. They were divided into two groups. The group with RCLP consisted of 17 Kannada speaking children with RCLP in the age range of 7 years to 13 years (seven females and ten males) with no hard and soft palate fistulas, having normal hearing threshold were included in the study. Children with any syndromes or associated abnormalities were excluded from the study. All these children were availing the speech evaluation and therapeutic services at a government institute. The control group consisted of 32 (15 females and 17 males) age and gender matched typically developing children (TDC) who were screened by speech language pathologist to rule out any sensory or neurological deficits and other abnormalities.

\section{Materials}

The phonation of vowels /a/ and /i/ were considered as stimuli to evaluate the perturbation measures in children with RCLP and TDC.

\section{Instrumentation}

An omni-directional distortion free I BALL microphone connected to a computer equipped with PRAAT 5.1 software [16] was used to record the stimuli and to measure the local jitter \%, local shimmer \% and Harmonic to noise ratio (HNR).

\section{Procedure}

The participants were seated comfortably in a sound treated room in an upright position. The I ball unidirectional microphone was positioned in front of the participant within $15 \mathrm{~cm}$ distance. Participants were instructed to sit relaxed and take a deep breath before they phonate vowel /a/ and /i/. Then clinician demonstrated and encouraged the participant to produce prolonged steady state vowels /a/ and /i/ at comfortable loudness level. Two repetitions of each vowel were done and the mean of the repetitions was calculated and documented. This is recorded using the PRAAT 5.1 software at a sampling rate of 44 $\mathrm{KHz}$ and saved for the further analysis.

\section{Data Analysis}

Computer software PRAAT 5.1 [16] developed at Phonetic Sciences, Department of the University of Amsterdam, was used for the voice analysis of the data collected. The steady state portion of the phonation around $4 \mathrm{sec}$ was selected using cursors for the analysis. Each vowel was analyzed using PRAAT to obtain 
voice report and the values of local jitter \%, local shimmer \%, and HNR were noted.

\section{Statistical Analysis}

Statistical analysis of the data was performed using parametric statistical tests. One way multivariate analysis (MANOVA) was performed to compare the jitter, shimmer, and HNR between the groups.

\section{Results}

The production of the vowel /a/ and / i/ by all the participants was recorded and analyzed to obtain local jitter \%, local shimmer
$\%$, and HNR values. The result indicated increased values of jitter and shimmer measures and reduced HNR for RCLP group than TDC as shown in Figure 2. The jitter values found to be more for the vowel /a/ than /i/ in RCLP group whereas it was more for /i/ in TDC. However, the differences were negligible. But for vowel /i/ the similar trend was not seen. Vowel /i/ had high shimmer and HNR values than /a/for both the groups. The results indicated statistically significant differences across the groups for local jitter \% of $/ \mathrm{a} /$ at the level of significance $\mathrm{p}<0.05$, and HNR of $/ \mathrm{i} /$ at the level of significance $\mathrm{p}<0.067$. The mean and standard deviation of these measures for the groups are mentioned in the below Table 1 .

Table 1: The mean and SD of jitter \%, Shimmer $\%$, and HNR across the groups.

\begin{tabular}{|c|c|c|c|c|c|c|c|c|}
\hline & \multicolumn{4}{|c|}{ Vowel /a/ } & \multicolumn{4}{|c|}{ Vowel /i/ } \\
\hline & \multicolumn{2}{|c|}{ RCLP } & \multicolumn{2}{|c|}{ TDC } & \multicolumn{2}{|c|}{ RCLP } & \multicolumn{2}{|c|}{ TDC } \\
\hline & Mean & S.D & Mean & S.D & Mean & S.D & Mean & S.D \\
\hline Local Jitter \% & 00.47 & 00.28 & 00.28 & 00.13 & 00.44 & 00.26 & 00.32 & 00.24 \\
\hline $\begin{array}{c}\text { Local } \\
\text { Shimmer \% }\end{array}$ & 07.99 & 03.88 & 06.49 & 03.58 & 08.90 & 04.24 & 07.60 & 03.11 \\
\hline HNR & 13.45 & 04.48 & 14.34 & 03.34 & 14.02 & 05.50 & 16.78 & 03.42 \\
\hline
\end{tabular}

SD: Standard Deviation, HNR: Harmonic to Noise Ratio, RCLP: Repaired Cleft Lip and Palate, TDC: Typically Developing Children
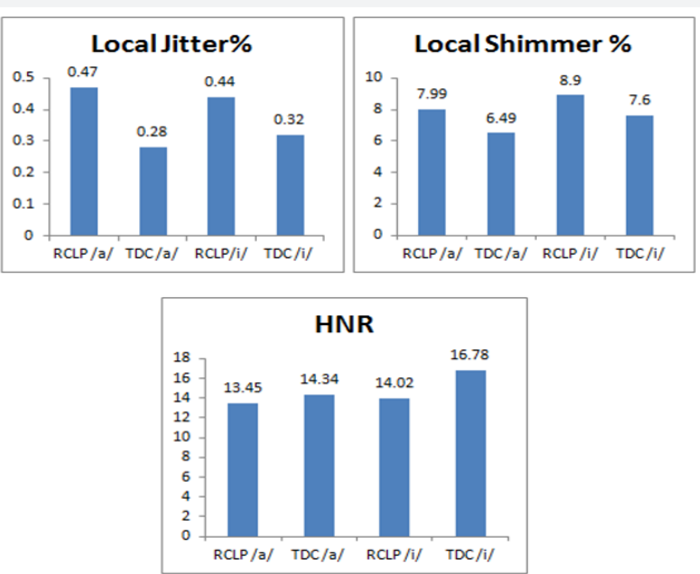

Figure 2: Mean jitter, shimmer and HNR measures across the groups and stimuli.

RCLP: Repaired Cleft Lip and Palate, TDC: Typically Developing Children, HNR: Harmonic to Noise Ratio

\section{Discussion}

The present study aimed to explore and compare the voice perturbation measures (jitter, shimmer, and harmonic -to-noise ratio) in children with RCLP and TDC. The results indicated several points of interest. Overall the results indicated relatively increased jitter, shimmer and reduced HNR measures in children with RCLP than TDC for both the stimuli (/a/ \& /i/). The voice perturbations are the cycle to cycle variations in the frequency (jitter) and intensity (shimmer) as a result of random neuromuscular and aerodynamic events happening while voicing which is evident in normal voice production also. However, the results of the present study indicated increased jitter and shimmer measures in children with RCLP compared to jitter and shimmer measures in TDC. This could be due to the abnormal oronasal coupling is seen during the production of oral consonants which can influence the changes in voice production in children with RCLP. The opening of the velopharyngeal valve provides two alternative pathways for the air to flow and this can alter the glottal volume velocity (flow rate) and transglottal pressure. The chidren with VPD always try to regulate the air flow by compensating at the laryngeal level i.e., the increase in the glottal resistance can regulate the subglottal pressure and decrease the flow of air to sustain the phonation for a long duration.

The increased perturbation measures could also be the result of hyper functional usage of voice to compensate for the loss of oral air pressure in the presence of VPD in children with RCLP. The study by Bzoch (1979) reported that the voice of children with cleft lip and palate is perceived to be weak and aspirated. Hence to compensate for the loudness level while speaking children with RCLP tend to use excessive phonatory effort resulting changes in the vocal fold movements. These changes result in altering the perturbation measures. Similar findings were reported by Zajac and Linville [4,17] indicating significant changes in the perturbation measures as a result of altered laryngeal physiology in the presence of VPD.

Another interesting aspect observed in the study is relatively reduced harmonic to noise ratio in children with RCLP than TDC. In the harmonic to noise ratio, the first component (harmonics) arises from the vibration of the vocal cords and the second (noise) follows from the glottal noise and is expressed in $\mathrm{dB}$. The evaluation of the two components reflects the efficiency of speech, i.e., the greater the flow of air expelled from the lungs into the energy of vibration of vocal cords. A voice sound 
is thus characterized by a high HNR, which is associated with sonorant and harmonic voice. A low HNR denotes an asthenic voice and dysphonia [14]. In the present study the HNR values of children with RCLP are found to be relatively less than TDC could be because of incoordination between the respiratory and laryngeal mechanism. Similar findings were also reported by Teixeira, Oliveira and Lopes [18] and the authors attributed to the imbalanced production of voice with the abnormal usage of resonance and laryngeal mechanism.

\section{Conclusion}

The study evaluated the salient features of voice characteristics in children with RCLP and compared with the typically developing children. The jitter, shimmer and HNR measures obtained in the study were still within the normative range as reported in the literature. However, it is interesting to note that jitter and shimmer measures were relatively increased and HNR measures were reduced in children with RCLP than TDS across the stimuli. This further helps to plan for detail physiological assessment and to plan for further intervention.

\section{Reference}

1. Fletcher SG, Adams LE, McCutcheon MJ (1989) Cleft palate speech assessment through oral-nasal acoustic measures. In Communicative Disorders Related to Cleft Lip and Palate. KR Bzoch (eds.). Little Brown, Boston, USA, 246-257.

2. Dalston RM, Seaver EJ (1992) Relative values of various standardized passages in the nasometric assessment of patients with velopharyngeal impairment. Cleft Palate-Craniofac J 29(1): 17-21.

3. Hocevar-Boltezar I, Jarc A, Kozelj V (2006) Ear, nose and voice problems in children with orofacial clefts. J Laryngol Otol 120(4): 276-281.

4. Zajac DJ, Linville RN (1989) Voice perturbations of children with perceived nasality and hoarseness. Cleft Palate J 26: 226-232.

5. Curtis J (1968) Acoustics of speech production and nasalization. In. Cleft Palate and Communication, Spriesterbach D, Sherman D (eds.), Academic Press, New York, USA, 1968: 27.

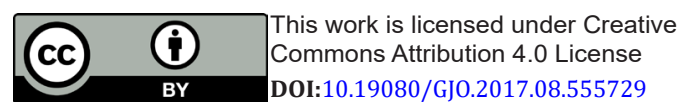

6. Sherman D, Spriestersbach DC, Noll JD (1959) Glottal stops in the speech of children with cleft palates. J Speech Hear Disord 24: 37-42.

7. John A, Sell D, Sweeney T, Harding-Bell A, Williams A (2006) The cleft audit protocol for speech-augmented: validated and reliable measure for auditing cleft speech. Cleft Palate Craniofac J 43: 272-288.

8. Warren DW, Duany L, Fischer N (1969) Nasal pathway resistance in normal and cleft lip and palate subjects. Cleft Palate J 6: 134

9. Hamlet S (1973) Vocal compensation: an ultrasonic study of vocal vibration in normal and nasal vowels. Cleft Palate J 10: 267-285.

10. Zwetsch I, Fagundes R, Russomano T, Scolari D (2006) Digital signal processing in the differential diagnosis of beningn larynx diseases. Porto Alegre 16(3).

11. Guimaraes IA (2007) Ciencia e a Arte de Voz Humana. Escola Superior de Saude de Alcoitao.

12. Yumoto E, Gould WJ, Baer T (1982) Harmonics-to-noise ratio as an index of the degree of hoarseness. J Acoust Soc of Am 71: 1544-1550.

13. Murphy P, Akande O (2005) Cepstrum-Based Estimation of the Harmonics-to-noise Ratio for Synthesized and Human Voice Signals. In Nonlinear Analyses and Algorithms for Speech Processing. LNAI 3817, Springer, Barcelona, UK.

14. Boersma P (1993) Accurate short-term analysis of the fundamental frequency and the harmonic-to-noise ratio of a sample sound. IFA. Proceedings 17: 97-110.

15. Hess DA (1959) Pitch, intensity and cleft palate voice. J Speech Lang Hear Res 2: 113-125.

16. Boersma P, Weenink D (2005) Praat: doing phonetics by computer. Phonetic Sciences, University of Amsterdam.

17. Aiswarya LV, Jayashree SB (2012) Perturbation in voice of children with cleft lip and/or palate. Advances in life science and technology 6: $52-56$.

18. Teixeira JP, Oliveira C, Lopes C (2013) Vocal acoustic analysis-jitter, shimmer and HNR parameters. Presented in an international conference on Health and Social Care Information Systems and Technologies, HCIST, Procedia Technology 9: 1112-1122.

\section{Your next submission with Juniper Publishers} will reach you the below assets

- Quality Editorial service

- Swift Peer Review

- Reprints availability

- E-prints Service

- Manuscript Podcast for convenient understanding

- Global attainment for your research

- Manuscript accessibility in different formats

( Pdf, E-pub, Full Text, Audio)

- Unceasing customer service

Track the below URL for one-step submission https://juniperpublishers.com/online-submission.php 